\title{
An Evaluation of Antibiotic Profile, Molecular Characterization and Risk Factors Associated with Carbapenem Resistant Non Fermentative Gram Negative Isolates in a Tertiary Care Centre
}

\author{
N. Grover ${ }^{1}$, N.K. Das ${ }^{2 *}$, M. Kumar ${ }^{3}$, R. Sriram ${ }^{1}$, V.L. Dudhat ${ }^{4}$, S. Prasanna ${ }^{5}$ and P. Pandit ${ }^{6}$ \\ ${ }^{1}$ Department of Microbiology, Armed Forces Medical College, Pune, Maharashtra, India \\ ${ }^{2}$ Department of Microbiology, Dr D Y Patil Vidyapeeth Medical College, \\ Pimpri, Pune-411018, India \\ ${ }^{3}$ Department of Lab Sciences \& Molecular Medicine, Army Hospital R \& R, Delhi, India \\ ${ }^{4}$ Department of Microbiology, Microbiology and HIC Sahyadri Speciality labs, Pune, India \\ ${ }^{5}$ Department of Microbiology, Shri Sathya Sai Medical College and Research Institute, \\ Ammapettai, Kancheepuram- 608103, India \\ ${ }^{6} \mathrm{Gd}$ Spl (Microbiology) Command Hospital, Kolkata, India \\ *Corresponding author
}

\author{
A B S T R A C T
}

\section{Keywords}

Antibiotic profile,

Molecular

characterisation,

Carbapenem

resistant non

fermentative Gram

negative bacteria

Article Info

Accepted:

12 April 2017

Available Online:

10 May 2017
Non-fermentative Gram negative bacteria (NFGNB) can cause serious infections in hospitalised patients. There has been an increase in resistance to carbapenems which is worrying as they are considered as antibiotics of last resort. Carbapenemases are responsible for carbapenem resistance. Study was undertaken to evaluate antibiotic profile, to ascertain risk factors associated and to detect genes responsible for carbapenem resistance in NFGNB isolates from acute wards of a tertiary care centre. The study was carried out in an urban tertiary care centre. Samples were collected from patients of acute wards and relevant clinical history was collected. Imipenem resistance detection and antibiotic susceptibility was done. A multiplex PCR was done on imipenem resistant isolates for detection of resistant genes. A total of 296 isolates were collected. Acinetobacter baumannii (132) followed by Pseudomonas aeruginosa (121) were the predominant isolates. OXA-51(72) and NDM were the predominant genes detected in Imipenem resistant A. baumannii and Pseudomonas aeruginosa (39). The carbapenem resistance in NFGNB in our hospital setting is mostly because of VIM, NDM, OXA-23, OXA-51. Constant monitoring of the incidence of such organisms in critical areas of the hospital, prompt recognition and getting rid of them is the only important preventive strategy.

\section{Introduction}

Among the non-fermentative Gram negative bacilli (NFGNB), Pseudomonas aeruginosa is considered a major pathogen; however in recent years other non-fermenters have also caused serious infections that place hospitalised patients at serious risk largely because of high intrinsic antibiotic resistance in these bacteria (Hancock, 1998; Su et al., 2009). Non-fermenters are generally multidrug resistant, with an increase in resistance to oxyimino-cephalosporins and carbapenems in the last two decades. Resistance not only compromises treatment but also leads to increased mortality, and inflated cost in hospitals (McGowan, 2006; Slama, 2008).

Carbapenems are stable to most $\beta$-lactamases including AmpC $\beta$-lactamases and extended spectrum $\beta$-lactamases (ESBL). Hence carbapenems are used as antibiotics of last 
resort for treating infections due to multidrugresistant Gram negative bacteria (Zhanel et al., 2007; Lee et al., 2003). Carbapenemases are enzymes secreted by bacteria which are relatively new and they have the ability to spread very rapidly. They confer resistance to the carbapenems as well as extended spectrum cephalosporins. There are various systems to classify them. According to the Ambler classification scheme, carbapenemases fall into class A (KPC, SME, NMC-A, IMI, GES), class B (IMP, VIM, NDM), and D (OXA enzymes) (Paterson et al., 2005). Carbapenemases are spreading throughout the world as the genes for most carbapenemases are plasmid mediated and are located on mobile cassettes inserted on variable regions in integrons resulting in enhanced potential for expression and dissemination (Henry et al., 2011). Identification and detection of carbapenemases producing organisms will guide the hospital infection control committee in preventing spread of multidrug resistant isolates and can quickly detect any outbreak of these organisms in critical care settings of hospital.

\section{Materials and Methods}

The study was carried out in the Department of Microbiology, of an urban tertiary care centre of western Maharashtra from Dec 2012 to Jul 2014 after institutional ethical committee clearance. Consecutive, non-repeat isolates of NFGNB were collected from clinical samples from inpatients of acute wards of a tertiary care centre. Detailed clinical history was recorded.

\section{Sample processing}

The clinical samples were processed and speciation of isolates was done by standard laboratory protocols (Collee et al., 2011; Govan et al., 2011).

\section{Antibiotic susceptibility testing}

Screening for carbapenem resistant NFGNB from the routine clinical samples was done by using $10 \mu \mathrm{g}$ imipenem discs (Fig. 1). Antibiotic susceptibility testing was performed on all NFGNB isolates by using Kirby-Bauer disc diffusion method as per CLSI guidelines 2012 (Fig. 2 and 3) (CLSI, 2012).

\section{Genotypic methods}

The presence of genes responsible for carbapenemases production like KPC, MBLs(VIM, NDM) and Oxacillinases OXA48, OXA-23, OXA-24, OXA-51, OXA-58 was done by PCR. In house strains were used as positive and negative controls.

\section{Primers and cycling conditions}

Primers used for detection of OXA-23, OXA24, OXA-51 and OXA-58 were referenced from a study by Huang et al., and for NDM, KPC, VIM and OXA-48 by Van der Zee et al., Monoplex PCR was performed on all isolates that were positive by multiplex PCR to differentiate between (OXA-23 and OXA51), (NDM and VIM) (Fig. 4 and 5).

\section{Statistical analysis}

Data in the present study was entered into spreadsheet (Excel 2007; Microsoft) for analysis. Unpaired student's t-test was used to measure test of significance for quantitative variables and Chi-square test for qualitative variables. Yate's correction was applied to the Chi-square test whenever frequency of variable was less than 5. All tests were two tailed and a $p$ value $<0.05$ was taken as "Significant". All tests were done using online GraphPad software:

http://www.graphpad.com/quickcalcs/conting ency $2 /$ and http://www.graphpad.com/quickcalcs/ttest2/ 


\section{Results and Discussion}

A total of 296 isolates of NFGNB were collected. Most common isolate collected during the present study was Acinetobacter baumannii (132) followed by Pseudomonas aeruginosa (121), Stenotrophomonas maltophilia (20), Alkaligenes faecalis (8), Burkholderia cepacia (4), Sphingomonas paucimobilis (4), Achromobacter denitrificans (2), Pseudomonas fluorescens (2), Pseudomonas putida (2) and Burkholderia pseudomallei (1). NFGNB was most commonly isolated from tracheal aspirate (101) followed by blood (76), pus (51), urine (25), sputum (11), body fluids (13) and other miscellaneous samples (19). Most samples in the present study were received from ICU-surgery (128) followed by general surgery ward (58), ICU-medicine (54), general medicine ward (24), general orthopaedics ward (14) and ENT ward (6).

94 isolates of A.baumannii were imipenem resistant. 63 isolates of P.aeruginosa were imipenem resistant. Thirty isolates of nonfermenters other than A.baumannii and P.aeruginosa were imipenem resistant. More than $90 \%$ of imipenem resistant isolates of $A$. baumannii (IRAB) were resistant to most other antibiotics. Piperacillin was resistant in all isolates. Polymixin B and Colistin were sensitive in $95.74 \%$ and $91.48 \%$ of IRAB.

More than $90 \%$ of isolates of imipenem resistant Pseudomonas aeruginosa (IRPA) were resistant to most antibiotics. Piperacillin was resistant in all imipenem resistant isolates. $95.23 \%$ of IRPA were sensitive to aztreonam. Polymixin B and Colistin were sensitive in $98.41 \%$ and $96.82 \%$ of IRPA isolates.

\section{PCR}

Out of 94 IRAB, OXA-51 was present in 72 of isolates, OXA-23 in 62, NDM in 56 and VIM in 26 isolates. 42 isolates had OXA-23, OXA-51 and NDM combination. KPC, OXA24, OXA-48, OXA-58 was not detected in any IRAB isolate. In 14 IRAB isolates no resistance genes was detected.

Out of 63 IRPA isolates NDM was present in 39 , VIM in 33 and OXA-48 in 5 isolate. OXA-23, OXA-24, OXA-51, OXA-58 and KPC were not detected in any isolate. In 16 isolates no genes under study were detected.

PCR was negative for any gene under study in non-fermenters other than A. baumannii and $P$. aeruginosa.

\section{Risk factor assessment (Table 1)}

Overall 72 patients died out of 296 patients harbouring NFGNB. Fifty one patients died from whom imipenem resistant strains were isolated. However there was no statistical significance seen in death of patients between imipenem sensitive and resistant isolates.

The mean duration of hospital stay in IRAB was 33.22 days and in ISAB was 22.11 days. The difference in mean duration of hospital stay in cases of IRAB and ISAB was not statistically significant. The mean duration of hospital stay in IRPA was 39.10 days and 22.38 days in ISPA. The difference in mean duration of hospital stay in ISPA and IRPA was statistically significant with a $p$ value of 0.0121 .Previous history of hospitalisation was seen in 33 patients infected with IRAB and in 12 patients with ISAB. The difference was not found to be statistically significant. Previous history of hospitalisation was seen in 32 patients infected with IRPA and in 19 patients with ISPA. The difference was found to be statistically significant with a $p$ value of 0.0447

Surgical intervention was there in 57 patients infected with IRAB and in 15 patients with 
ISAB patients. The difference was found to be statistically significant with a $p$ value of 0.0270 . Surgical intervention was seen in 40 patients infected with IRPA and in 25 patients with ISPA patients. It was statistically significant with a $p$ value of 0.0246 .

39 patients infected with IRAB and 10 patients infected with ISAB had mechanical ventilation. The difference was not found to be statistically significant. Intervention of mechanical ventilation was found to be a statistically significant risk factor for infection with IRPA with a $p$ value of 0.0490 . Treatment with carbapenems earlier in course of disease or in recent past was not found to be a significant risk factor.

Fifty seven patients with IRAB and 12 patients with ISAB were immunocompromised. This was found out to be a significant risk factor with a $p$ value of 0.0243. 44 patients with IRPA and 29 patients with ISPA infection were immunocompromised. This was statistically significant with a $p$ value of 0.0258 .

The burden of infectious diseases is among the highest in India making the treatment with antibiotics play a huge role in determining mortality and morbidity (Choudhury et al., 2012). Ease of mobility by human due to travel, allow different bacterial plasmid and clones to be transported to different countries. Selection pressure for carbapenem resistance is a major concern as only a few antibiotics are there which are reserved for resistance beyond the carbapenems. Most isolates however still are sensitive to colistin and tigecycline.

A total of 296 samples were collected during the study period. The most common isolate was Acinetobacter baumannii, followed by Pseudomonas aeruginosa. Imipenem resistance was seen in $63.1 \%$ of total isolates.
Literatures from SE Asia mention prevalence of carbapenem resistance in non-fermenters varying from 36 to $90 \%$ (Goel et al., 2011). Considering sample wise distribution, most samples from which non-fermenters were isolated were those from tracheal aspirate (34.12\%) followed by blood $(25.67 \%)$, pus $(17.22 \%)$, and urine $(8.44 \%)$. Amudhan et al., had predominant NFGNB isolates from respiratory secretions (Amudhan et al., 2012). Kalidas et al., isolated non-fermenters predominantly from pus (Rit et al., 2013).

$71.21 \%$ of Acinetobacter baumannii were imipenem resistant. Most of these IRAB isolates were from ICU-surgery followed by ICU-medicine and surgery ward. This is similar to the finding by Baran et al., (2008) who found IRAB more in ICUs than in wards and Khajuria et al., (2014) who reported that among his imipenem resistant isolates most isolates were from ICU-surgery.

52.06\% of Pseudomonas aeruginosa were imipenem resistant. Bhalerao, Behera and Onguru et al., (2010; 2008; 2008) have reported imipenem resistance in $P$. aeruginosa at $67.5 \%, 69 \%$ and $44.1 \%$ respectively. Most IRPA came from ICUsurgery $(39.68 \%)$ followed by ICU-medicine and surgery wards (20.63\%).

Among IRAB, $76.59 \%$ of isolates were positive for OXA-51, 65.95\%were OXA-23 positive. Khajuria et al., (2014) had $44.76 \%$ of his isolates positive for OXA-51 and $52.38 \%$ were positive for OXA-23. His study also found OXA-58, OXA-24 in his isolates, which were lacking in our study. $9.5 \%$ isolates were both OXA-23 and OXA-51 positive. $44.68 \%$ isolates were positive for OXA-23, OXA-51 and NDM. $1.06 \%$ of isolates were positive for only NDM or VIM. Total $27.65 \%$ of isolates were positive for VIM. Amudhan et al., (2012) reported $45.68 \%$ of resistant isolates to harbour VIM. 
11.7\%isolates were positive for OXA-23, OXA-51, NDM and VIM. Niranjan et al., reported that OXA-51, VIM-1 and IMP-1 were present in all isolates of A. baumannii. They also found OXA-23 in $46.66 \%$ of isolates and no isolate was positive for OXA24 and OXA-58 (Niranjan et al., 2013). In our study also multiple resistance genes were seen to be present in a single isolates and there was presence of MBL genes with class $\mathrm{D}$ carbapenemases and none of the isolates were positive for OXA-24 and OXA-58. In the present study we found NDM in 59.57\% of imipenem resistant isolates whereas Niranjan et al., found NDM in about 30\% isolates and Farzana found 26.6\% of IRAB to be NDM. In all $71.27 \%$ of IRAB were MBL by molecular methods. Farzana et al., 2013 found all his IRAB as MBL producers. The presence of NDM emphasizes the instant reception of A.baumannii to carbapenemase genes. OXA-23 is present in class I integrons which can also carry genes for resistance to drugs like aminoglycosides and fluoroquinolones. These integrons can be easily transferred to other gram negative bacteria by "genetic capitalism". No isolate was positive for OXA-24, OXA-58, KPC and
OXA-48. The isolates negative for test genes in PCR can have genes other than these test genes for carbapenem resistance. In IRPA, overall $61.9 \%$ of isolates were positive for NDM, 52.38\% for VIM and 7.9\% were OXA48 positive. $42.85 \%$ had both NDM and VIM, $14.28 \%$ of isolates were only NDM positive and $9.52 \%$ were only VIM positive. Farzana detected NDM in $18.75 \%$ of IRPA isolates. Chaudhary et al., (2014) reported the presence of OXA-48 in their carbapenem resistant P.aeruginosa isolates. We found $7.9 \%$ of isolates to be positive for OXA-48. $25.39 \%$ of IRPA isolates were negative for any resistance genes tested. In imipenem resistant isolates of A.baumannii it was seen that most isolates were resistant to first line antibiotics. However the isolates were consistently susceptible to colistin and polymixin Khajuria et al., and Shivaprasad et al., found that most of their isolates were susceptible to tigecycline and colistin. The same observation was seen in case of P.aeruginosa in which also most of the first line antibiotics were resistant over 90\% except for polymixin B, colistin, and aztreonam.

Table.1 Comparison between imipenem sensitive and resistant isolates considering various risk factors

\begin{tabular}{|l|c|c|c|c|c|c|}
\hline \multicolumn{1}{|c|}{ Risk factors } & $\begin{array}{c}\text { No of } \\
\text { IRAB }\end{array}$ & $\begin{array}{c}\text { No of } \\
\text { ISAB }\end{array}$ & $\begin{array}{c}\boldsymbol{p} \\
\text { value }\end{array}$ & $\begin{array}{c}\text { No of } \\
\text { IRPA }\end{array}$ & $\begin{array}{c}\text { No of } \\
\text { ISPA }\end{array}$ & $\begin{array}{c}\boldsymbol{p} \\
\text { value }\end{array}$ \\
\hline $\begin{array}{l}\text { Mean duration of } \\
\text { hospital stay (SD) }\end{array}$ & $\begin{array}{c}33.22 \\
(41.09)\end{array}$ & $\begin{array}{c}22.11 \\
(22.61)\end{array}$ & 0.1182 & $\begin{array}{c}39.10 \\
(41.05)\end{array}$ & $\begin{array}{c}22.38 \\
(29.61)\end{array}$ & $\mathbf{0 . 0 1 2 1}$ \\
\hline $\begin{array}{l}\text { Previous H/O } \\
\text { hospitalization }\end{array}$ & 33 & 12 & 0.6987 & 32 & 19 & $\mathbf{0 . 0 4 4 7}$ \\
\hline Surgical intervention & 57 & 15 & $\mathbf{0 . 0 2 7 0}$ & 40 & 25 & $\mathbf{0 . 0 2 4 6}$ \\
\hline $\begin{array}{l}\text { Mechanical } \\
\text { ventilation history }\end{array}$ & 31 & 7 & 0.0944 & 15 & 11 & 0.5169 \\
\hline $\begin{array}{l}\text { Treatment } \\
\text { with carbapenems }\end{array}$ & 57 & 12 & $\mathbf{0 . 0 2 4 3}$ & 44 & 29 & $\mathbf{0 . 0 2 5 8}$ \\
\hline $\begin{array}{l}\text { Immunocompromised } \\
\text { status }\end{array}$ & & & 0.1023 & 22 & 11 & $\mathbf{0 . 0 4 9 0}$ \\
\hline
\end{tabular}


Fig.1 Imipenem resistant Pseudomonas aeruginosa

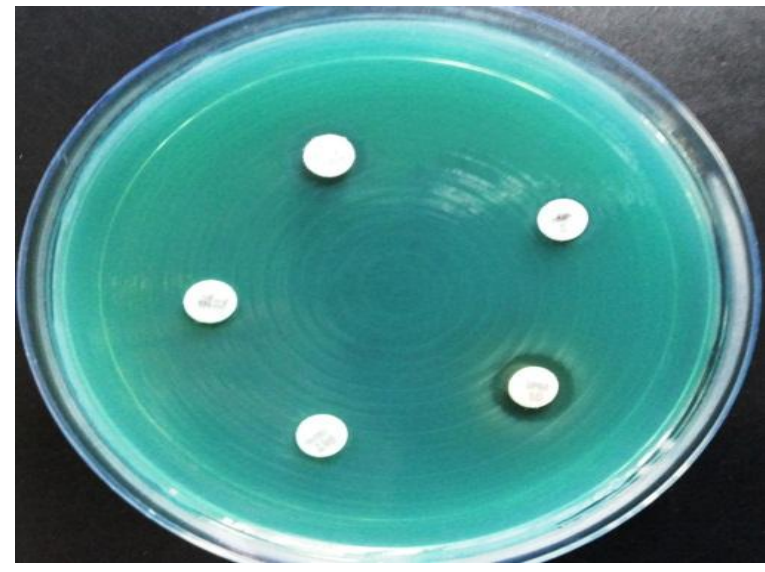

Fig.2 Antibiotic profile of imipenem resistant A. baumannii

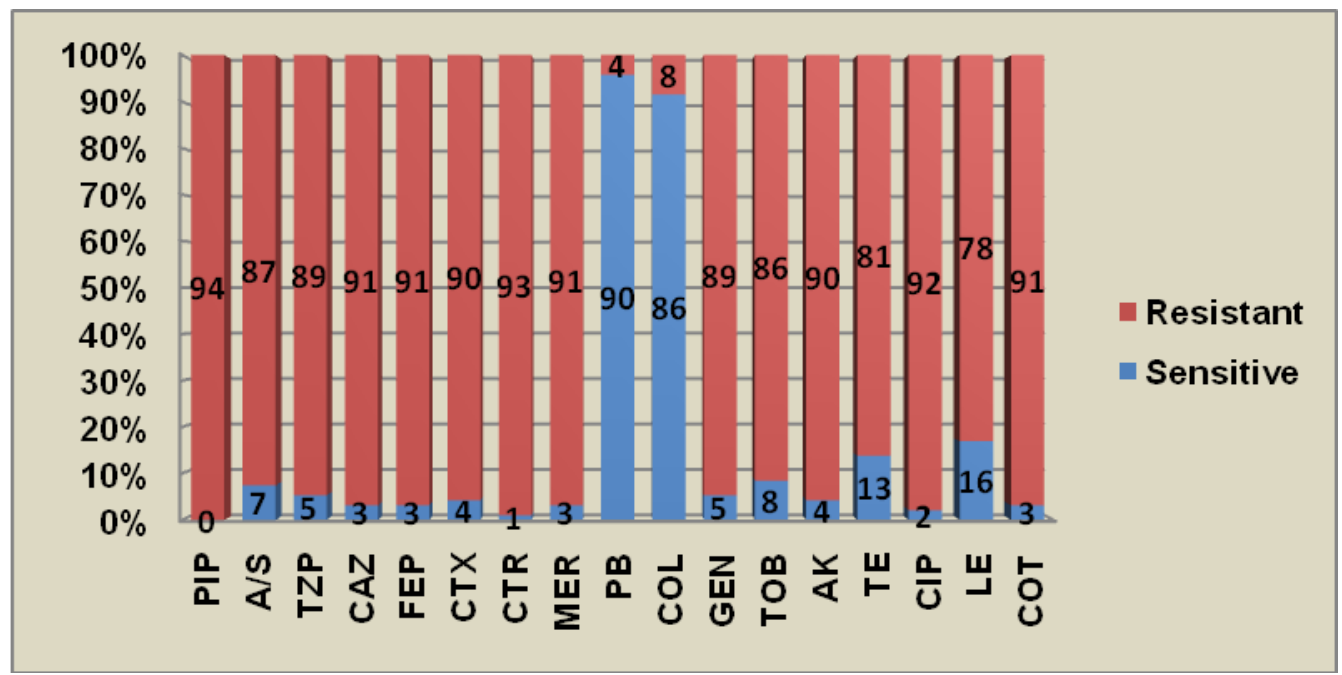

Fig.3 Antibiotic profile of imipenem resistant Pseudomonas aeruginosa

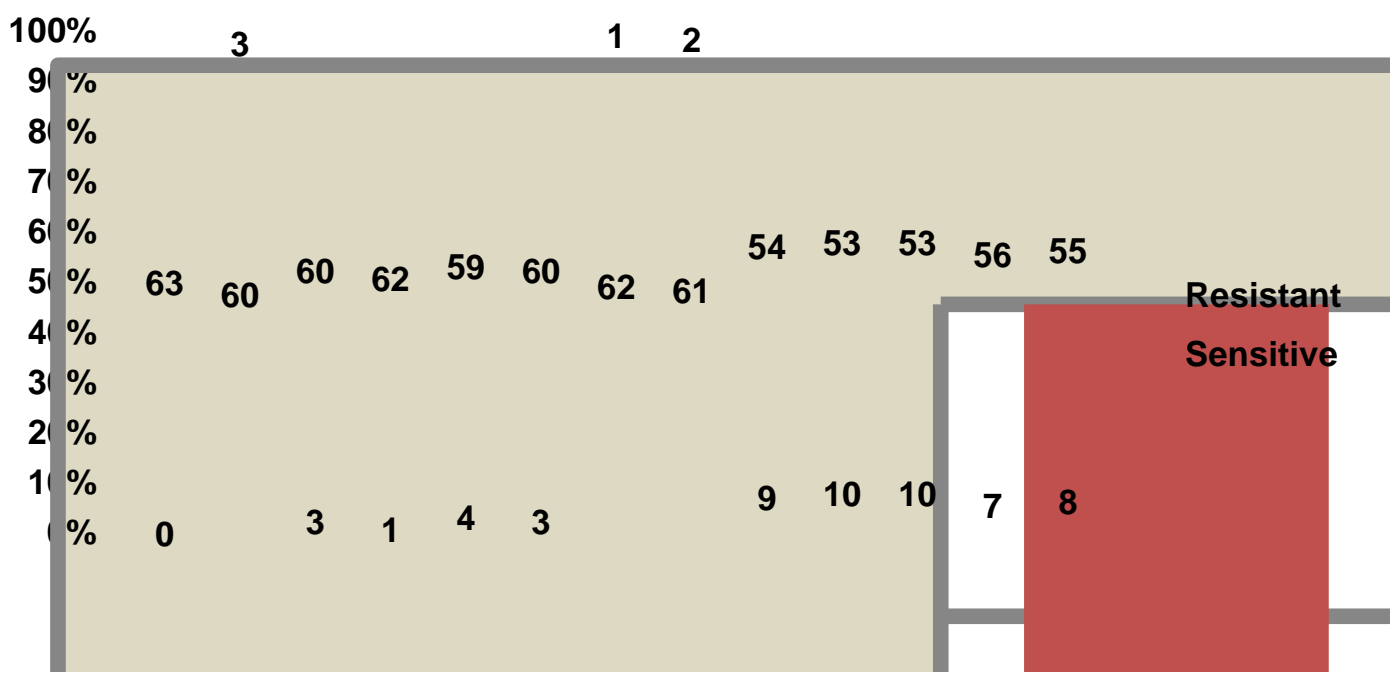


Fig.4 Gel electrophoresis result showing OXA-23 (116 bp), OXA-51 (112 bp), MW-Molecular marker, NC-negative control, PC-positive control

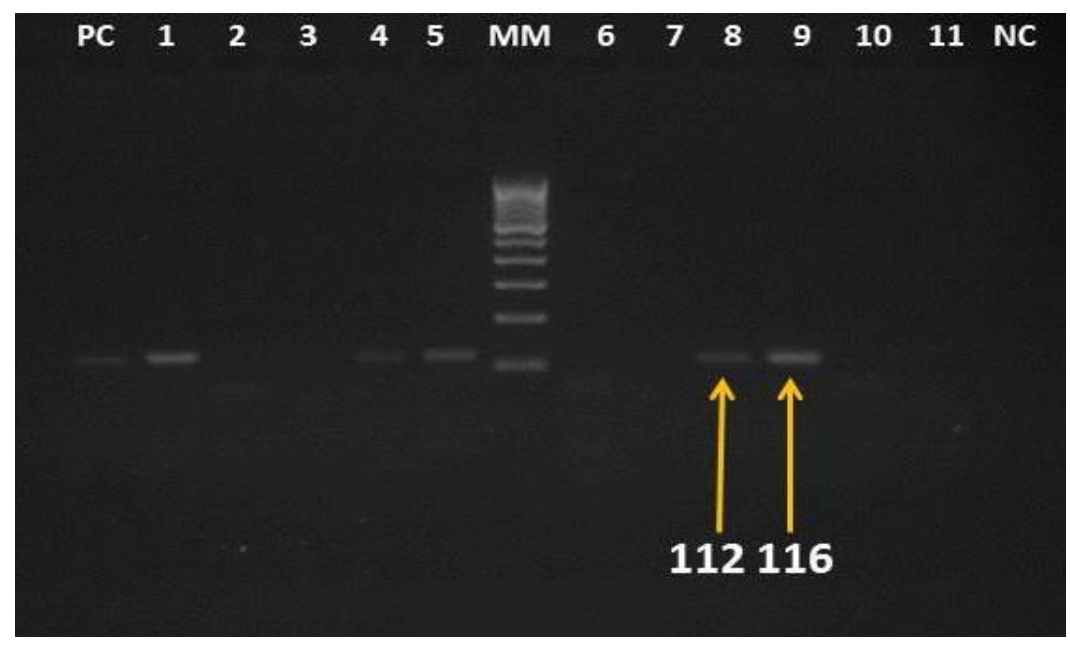

Fig.5 Gel electrophoresis result showing NDM (83bp), VIM (92bp), OXA-48 (438 bp) MMMolecular marker, NC-negative control

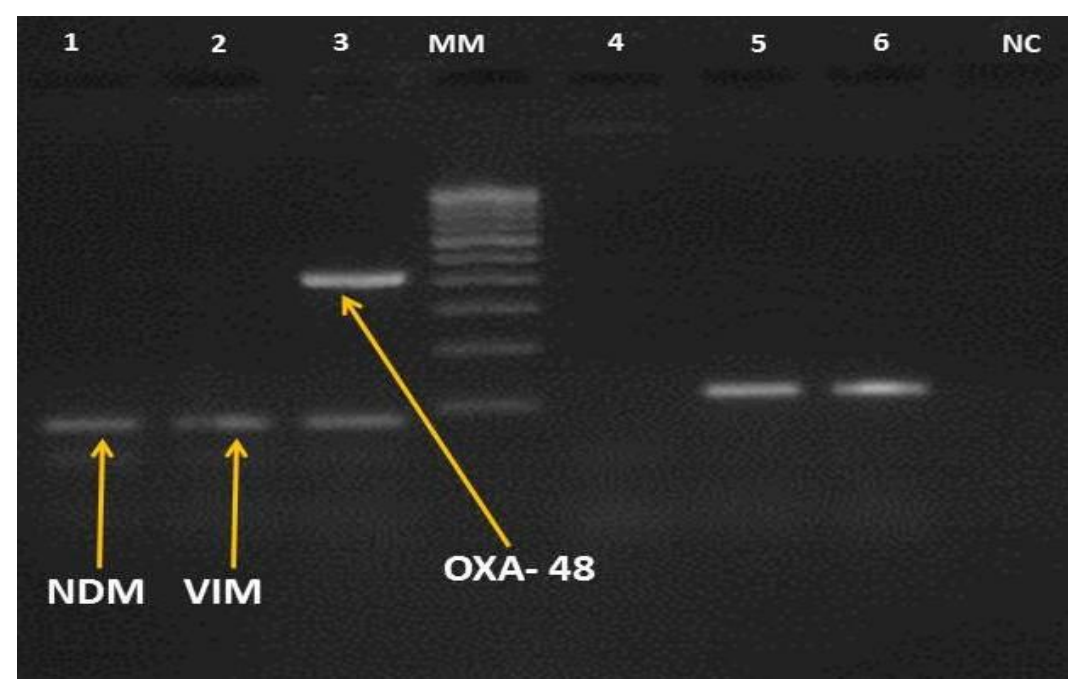

Onguru et al., (2008) reported the presence of more than $70 \%$ resistance to most antibiotics in IRPA. Amudhan et al., and Behera et al., found that $91.8 \%$ and $87.5 \%$ of their imipenem resistant $P$. aeruginosa isolates were polymixin $\mathrm{B}$ sensitive. Colistin and polymixin $\mathrm{B}$ are considered as drugs of last resort in carbapenem resistant NFGNB hence should be used judiciously (Al-Anazi, 2014).

The patients harbouring IRPA isolates had a significant increase in duration of stay in hospital. Onguru et al., (2008) reported that longer duration of hospital stay was associated with infection with IRPA.

Previous history of hospitalisation was seen as a significant finding for infection with IRPA infection. Zavascki and Harris et al., (2005; 2002) reported hospitalisation in the previous year as a significant risk factor for infection with IRPA. History of surgical 
intervention was found to be a significant finding for harbouring IRAB and IRPA infection. Baran reported that surgical intervention was significantly associated with infection with IRAB infection. Mechanical ventilation was seen to be associated with IRPA infection. Zavascki et al., (2005) had mechanical ventilation as a significant risk factor for infection with IRPA. Immunocompromised state was found to be a significant risk factor for IRAB and IRPA infection.

Simple methods like adherence to hand hygiene practices, suction and ventilator decontamination and environmental cleaning can go a long way to reduce containment of infections. Hence consensus and co-operation among hospital staff, strong infection control guidelines and antibiotic stewardship must be in place in critical care settings to prevent infection due to multidrug resistant bacteria

In conclusion, multiple mechanisms of carbapenem resistance are present in NFGNB. The carbapenem resistance mechanism in NFGNB in our hospital setting is mostly because of metallo- $\beta$-lactamases (VIM, NDM) and Oxacillinases enzyme (OXA-23, OXA-51 type). One or more types of mechanisms might be acting synergistically to cause high level carbapenem resistance. The carbapenem resistant NFGNB strains isolated in our hospital are mainly from ICU and these isolates are multi drug resistant. Since there are limited treatment options against these, continuous vigilance, early identification and treatment is very important to prevent further spread. Constant and regular monitoring of the incidence of such organisms in various critical areas of the hospital like ICU and acute medical units, prompt recognition of potential areas of colonisation and getting rid of them is the only important preventive strategy. To keep this problem in check, simple infection control measures like proper hand washing, adherence to infection control guidelines and antibiotic stewardship must be followed and time to time revision must be done.

\section{References}

Al-Anazi, K.A., Al-Jaseer. 2014. Infections caused by Acinetobacter baumannii in recipients of hematopoietic stem cell transplantation. Front Oncol., 4: 186.

Amudhan, M.S., Sekar, U., Kamalanathan, A., Balaraman, S. 2012. bla $a_{I M P}$ and bla $_{V I M}$ mediated carbapenem resistance in Pseudomonas and Acinetobacter species in India. J. Infect. Dev. Ctries., 6(11): 757-62.

Baran, G., Erbay, A., Bodur, H., Onguru, P., Akinci, E., Balaban, N., et al., 2008. Int. J. Infect. Dis., 12: 16-21.

Behera, B., Mathur, P., Das, A., Kapil, A., Sharma, V. 2008. An evaluation of four different phenotypic techniques for detection of metallo- $\beta$-lactamase producing Pseudomonas aeruginosa. Indian J. Med. Microbiol., 26(3): 233-7.

Bhalerao, D.S., Roushani, S., Kinikar, A.G., Akhter, I. 2010. Study of metallo-beta lactamase producing Pseudomonas aeruginosa in Pravara rural hospital. Pravara Med. Rev., 2(3): 16-9.

Chaudhary, M., Payasi, A. 2014. Prevalence genotyping of Escherichia coli and Pseudomonas aeruginosa clinical isolates for oxacillinase resistance and mapping susceptibility behaviour. $J$. Microb. Biochem. Technol., 6(2): 63-7.

Choudhury, R., Panda, S., Singh, D.V. Emergence and dissemination of antibiotic resistance: A global problem. Indian J. Med. Microbiol., 30: 384-90.

Chroma, M., Kolar, M. 2010. Genetic methods for detection of antibiotic resistance: Focus on Extended-spectrum $\beta$-lactamases. Biomed. Pap Med Fac Univ. Palacky Olomouc Czech Repub., 154(4): 289-96. 
CLSI. 2012. Performance standards for antimicrobial susceptibility testing; twenty-second informational supplement. CLSI document M100S22. Wayne, PA: Clinical and Laboratory Standards Institute.

Collee, J.G., Duguid, J.P., Fraser, A.G., Marmion, B.P., Simmons, A. 2011. Laboratory strategy in the diagnosis of infective syndromes. In: Collee JG, Fraser AG, Marmion BP, Simmons A, editors. Mackie \& McCartney Practical Medical Microbiology. $14^{\text {th }}$ ed. India: Churchilll Livingstone.

Farzana, R., Shamsuzzaman, S.M., Mamun, K.Z. 2013. Isolation and molecular characterisation of New Delhi metallo$\beta$-lactamase-1 producing superbug in Bangladesh. Emerging Problems in Infectious diseases, 7(3): 161-8.

Goel, N., Wattal, C., Oberoi, J.K., Raveendran, R., Datta, S., Prasad, K.J. 2011. Trend analysis of antimicrobial consumption and development of resistance in non-fermenters in a tertiary care hospital in Delhi, India. $J$. Antimicrob. Chemother., 60(7): 162530.

Govan, J.R.W. 2011. Pseudomonas, Stenotrophomonas, Burkholderia. In: Collee JG, Fraser AG, Marmion BP, Simmons A, editors. Mackie \& McCartney Practical Medical Microbiology. $14^{\text {th }}$ ed. India: Churchilll Livingstone.

Hancock, R.E.W. 1998. Resistance mechanism in Pseudomonas aeruginosa and other Non-fermentative Gramnegative bacteria. Clin. Infectious Dis., 27(suppl 1): S93-9.

Harris, A., Smith, D., Johnson, J., Bradham, D., Roghmann, M. 2002. Risk factors for imipenem resistant P.aeruginosa among hospitalised patients. Clin. Infect. Dis., 34: 340-5.
Henry, D.A., Speert, D.P. 2011. Pseudomonas. In: Versalovic J, Carroll KC, Funke G, Jorgensen JH, Landry ML, Warnock DW, editors. Manual of Clinical Microbiology. $10^{\text {th }}$ ed. Washington, DC: ASM Press.

Huang, X.Z., Cash, D.M., Chahine, M.A., Nikolich, M.P., Craft, D.W. 2012. Development and validation of a multiplex Taqman real-time PCR for rapid detection of genes encoding four types of class D carbapenemase in A.baumannii. J. Med. Microbiol., 61: 1532-7.

Khajuria, A., Praharaj, A.K., Kumar, M., Grover, N. 2014. Molecular characterisation of carbapenem resistant isolates of Acinetobacter baumannii in an intensive care unit of a tertiary care centre. J. Clin. Diag Res., 8(5): 38-40.

Lee, K., Lim, Y.S., Yong, D., Yum, J.H., Chong, Y. 2003. Evaluation of the Hodge Test and the Imipenem-EDTA Double-Disk Synergy Test for Differentiating Metallo- $\beta$-LactamaseProducing Isolates of Pseudomonas spp. and Acinetobacter spp. J. Clin. Microbiol., 4623-9.

McGowan, J.E. 2006. Resistance in nonfermenting Gram-negative bacteria: multidrug resistance to the maximum. Am. J. Med., 119 Suppl 1: S29-36.

Niranjan, D.K., Singh, N.P., Manchanda, V., Rai, S., Kaur, S. 2013. Multiple carbapenem hydrolysing genes in clinical isolates of Acinetobacter baumannii. Indian J. Med. Microbiol., 31(3): 237-41.

Onguru, P., Erbay, A., Bodur, H., Baran, G., Akinci, E., Balaban, N., et al., Imipenem resistant Pseudomonas aeruginosa: risk factors. J. Korean Med. Sci., 23: 982-7.

Paterson, D.L., Bonomo, R.A. 2005. Extended-spectrum $\beta$-lactamases: a 
clinical update. Clin. Microbiol. Rev., 18: 657-86.

Rit, K., Nag, F., Raj, H.J., Maity, P.K. 2013. Prevalence and susceptibility profiles of non-fermentative gram-negative bacilli infection in a tertiary care hospital of Eastern India. Indian J. Clin. Practice, 24(5): 451-5.

Shivaprasad, A., Antony, B., Shenoy, P., MBL \& Carbapenemase Production in A.baumannii. J. Clin. Diag. Res., 8(5): 5-8.

Slama, T.G. 2008. Gram-negative antibiotic resistance: there is a price to pay. Crit. Care, 12 Suppl 4: S4.

Su, S.C., Vaneechoutte, M., Dijkshoorn, L., Wei, Y.F., Chen, Y.L., Chang, T.C. 2009. Identification of non-fermenting Gram-negative bacteria of clinical importance by an oligonucleotide array. J. Med. Microbiol., 58(5): 596-605.

Van der Zee, A., Roorda, L., Bosman, G., Fluit, A.C., Hermans, M., Smits, P.H. et al. Multicentre evaluation of real-time multiplex PCR for detection of carbapenemase genes OXA-48, VIM, NDM and KPC. BMC Infectious Dis., 14(27): 1471-2334.

Zavascki, A.P., Cruz, R., Coldani, L. 2005. Risk factors for imipenem resistant Pseudomonas aeruginosa comparative analysis of two case control studies in hospitalised patients. J. Hosp. Infect., 59: 96-101.

Zhanel, G.G., Wiebe, R., Dilay, L., Thomson, K., Rubinstein, E., Hoban, D.J., et al. 2007. Comparative review of carbapenems. Drugs, Volume 67(7): 1027-52.

\section{How to cite this article:}

Grover, N., N.K. Das, M. Kumar, R. Sriram, V.L. Dudhat, S. Prasanna and Pandit, P. 2017. An Evaluation of Antibiotic Profile, Molecular Characterization and Risk Factors Associated with Carbapenem Resistant Non Fermentative Gram Negative Isolates in a Tertiary Care Centre. Int.J.Curr.Microbiol.App.Sci. 6(5): 1057-1066. doi: https://doi.org/10.20546/ijcmas.2017.605.115 